\title{
The activation of NLRP3 inflammasome potentiates the immunomodulatory abilities of mesenchymal stem cells in a murine colitis model
}

\author{
Ji-Su Ahn ${ }^{1, \#}$, Yoojin Seo ${ }^{2, \#}$, Su-Jeong $\mathrm{Oh}^{1}$, Ji Won Yang ${ }^{1}$, Ye Young Shin ${ }^{1}$, Byung-Chul Lee ${ }^{3}$, Kyung-Sun Kang ${ }^{3}$, \\ Eui-Suk Sung ${ }^{4}$, Byung-Joo Lee ${ }^{5}$, Hemn Mohammadpour ${ }^{6}$, Jin Hur ${ }^{7}$, Tae-Hoon Shin ${ }^{8, *}$ E Hyung-Sik Kim ${ }^{1,2, *}$ \\ ${ }^{1}$ Department of Life Science in Dentistry, School of Dentistry, Pusan National University, Yangsan 50612 , ${ }^{2}$ Dental and Life Science \\ Institute, Pusan National University, Yangsan 50612, ${ }^{3}$ Adult Stem Cell Research Center and Research Institute for Veterinary Science, \\ College of Veterinary Medicine, Seoul National University, Seoul 08826, ${ }^{4}$ Department of Otorhinolaryngology, Head and Neck Surgery, \\ Pusan National University Yangsan Hospital, Yangsan 50612, ${ }^{5}$ Department of Otorhinolaryngology-Head and Neck Surgery, Biomedical \\ Research Institute, Pusan National University Hospital, Pusan National University School of Medicine, Busan 49241, Korea, ${ }^{6}$ Department \\ of Immunology, Roswell Park Comprehensive Cancer Center, Buffalo, NY 14203, USA, ${ }^{7}$ Department of Convergence Medicine, Pusan \\ National University School of Medicine, Yangsan 50612, Korea, ${ }^{8}$ Translational Stem Cell Biology Branch, National Heart, Lung, and Blood \\ Institute, National Institutes of Health, Bethesda, MD 20892, USA
}

Inflammasomes are cytosolic, multiprotein complexes that act at the frontline of the immune responses by recognizing pathogen- or danger-associated molecular patterns or abnormal host molecules. Mesenchymal stem cells (MSCs) have been reported to possess multipotency to differentiate into various cell types and immunoregulatory effects. In this study, we investigated the expression and functional regulation of NLR Family Pyrin Domain Containing 3 (NLRP3) inflammasome in human umbilical cord blood-derived MSCs (hUCB-MSCs). hUCB-MSCs expressed inflammasome components that are necessary for its complex assembly. Interestingly, NLRP3 inflammasome activation suppressed the differentiation of hUCB-MSCs into osteoblasts, which was restored when the expression of adaptor proteins for inflammasome assembly was inhibited. Moreover, the suppressive effects of MSCs on T cell responses and the macrophage activation were augmented in response to NLRP3 activation. In vivo studies using colitic mice revealed that the protective abilities of hUCB-MSCs increased after NLRP3 stimulation. In conclusion, our findings suggest that the NLRP3 inflammasome components are expressed in hUCB-MSCs and its activation can regulate the differentiation capability and the immunomodu-

*Corresponding authors. Hyung-Sik Kim, Tel: +82-51-510-8231; Fax: +82-51-510-8210; E-mail: hskimcell@pusan.ac.kr; Tae-Hoon Shin, Tel: +1-301-827-0565; Fax: +1-877-645-2448; E-mail: taehoon.shin @nih.gov

"These authors contributed equally to this work.

https://doi.org/10.5483/BMBRep.2020.53.6.065

Received 31 March 2020, Revised 21 April 2020, Accepted 30 April 2020

Keywords: Colitis, Immunomodulation, Inflammasome, Mesenchymal stem cell, NLRP3 latory effects of hUCB-MSCs. [BMB Reports 2020; 53(6): 329-334]

\section{INTRODUCTION}

The mammalian immune system consists of innate and adaptive immunity, which cooperatively work for the elimination of exogenous pathogens or endogenous danger signals. The innate immune system eradicates the infective microbial pathogens upon their recognition and initiates further inflammatory responses (1-4). The innate immune cells possess various sensor proteins to distinguish self-molecules from foreign substances (5, 6). Pattern recognition receptors (PRRs) are sensors for pathogenassociated molecular patterns (PAMPs) (7). Toll-like receptors (TLRs) and Nod-like receptors (NLRs) are representative families of PRRs. Previous studies have reported that TLRs and NLRs are functionally expressed in mesenchymal stem cells (MSCs) (8-12). In our previous study, the stimulation of nucleotide-binding oligomerization domain 2 (NOD2) resulted in the altered differentiation capabilities or immunoregulatory abilities of MSCs (11). Inflammasome is the recently reported cytosolic protein complex that is involved in the initiation of the inflammatory responses in response to exogenous microbial infection and endogenous danger signals. Inflammasome generally contains three components: a cytosolic PRR that senses stimuli, the enzyme caspase- 1 which converts cytokine precursors into mature cytokines, and the adaptor protein such as apoptosis-associated speck-like protein containing a caspase recruitment domain (ASC) $(13,14)$. Assembly of the inflammasome leads to the activation of caspase- 1 which subsequently transforms the pro-inflammatory cytokines such as interleukin-1 $\beta$ (IL-1 $\beta$ ) and interleukin-18 (IL-18) to their mature and active forms (15).

The NLRP3 inflammasome has been well studied and most frequently implicated as a key player in the pathogenesis of

ISSN: 1976-670X (electronic edition)

Copyright (C) 2020 by the The Korean Society for Biochemistry and Molecular Biology

(c) This is an open-access article distributed under the terms of the Creative Commons Attribution Non-Commercial License (http://creativecommons.org/licenses/by-nc/4.0) which permits unrestricted non-commercial use, distribution, and reproduction in any medium, provided the original work is properly cited. 
various diseases. NLRP3 inflammasome is activated via two-step processes including priming step and activation step (16-18). Currently, little is known about the expression and role of the inflammasome in non-immune cells including adult stem cells.

MSCs have been reported to exhibit immunoregulatory properties against various autoimmune or allergic diseases, as well as differentiation potential into mesodermal lineages (19-25). This immunomodulatory effect of MSCs on immune cells is mostly exerted by the combination of soluble factors (26-32). In our previous findings, we showed that the priming of NOD2 receptors in MSCs before the administration led to the improved therapeutic efficacy against colitis and atopic dermatitis through regulation of $\mathrm{T}$ cells or mast cells, respectively, via the production of prostaglandin $\mathrm{E}_{2}\left(\mathrm{PGE}_{2}\right)(33,34)$. Although few studies including the one by $\mathrm{Oh}$ et al. revealed that MSCs can regulate the activation of NLRP3 inflammasome in macrophages or diseases (35), only one study has reported the expression of inflammasome components and their functions in MSCs (36). Moreover, in the reported study, changes in MSC function were solely focused on osteogenic and adipogenic differentiation after NLRP3 inflammasome activation. Therefore, in this study, for the first time in our knowledge, we sought to investigate whether hUCB-MSCs express NLRP3 inflammasome complexes and the activation of NLRP3 inflammasome has any influence on the immunoregulatory function of hUCB-MSCs.

\section{RESULTS}

Inflammasome components are expressed in hUCB-MSCs We first investigated the expression of inflammasome components, as well as final products of inflammasome activation including IL-1 $\beta$ in hUCB-MSCs. To activate NLRP3 inflammasome, LPS and ATP were treated for 4 hours and 45 minutes, respectively. In THP-1 cells, the expressions of NLRP3, cleaved caspase-1, and pro/mature IL-1 $\beta$ were elevated in the presence of LPS or LPS+ATP compared to the control group or ATPtreated group. hUCB-MSCs expressed NLRP3, ASC, caspase-1, and IL-1 $\beta$. Interestingly, LPS + ATP treatment in hUCB-MSCs increased the expression of NLRP3, cleaved caspase-1, and pro IL-1 $\beta$. However, the expression of mature IL-1 $\beta$ was not altered by LPS + ATP treatment (Fig. 1A). Subsequently, we measured the production of IL-1 $\beta$ from hUCB-MSCs after NLRP3 inflammasome activation. However, in hUCB-MSCs, NLRP3 activation did not induce the production of IL-1 $\beta$ and the basal level of IL-1 $\beta$ production was too low to exert physiological functions (Fig. 1B). Taken together, these findings indicate that hUCB-MSCs express the components of NLRP3 inflammasome and its activation is induced in a manner similar in the macrophages. However, the final product of the activation, mature IL-1 $\beta$, was not robustly secreted from hUCB-MSCs in response to NLRP3 activation, indicating that inflammasome activation in mesenchymal non-immune cells might serve different functions as reported in innate immune cells.
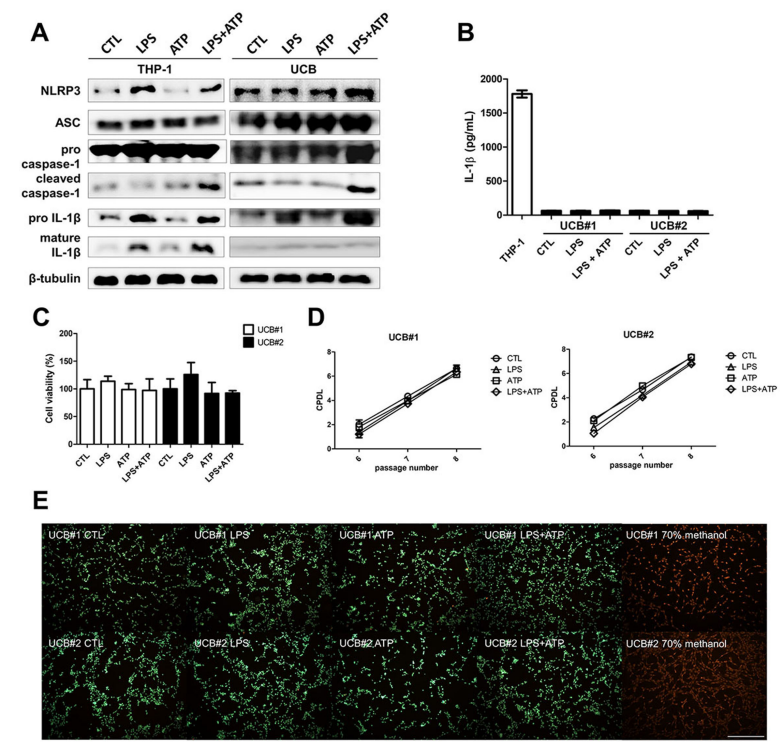

Fig. 1. The expression of inflammasome components and viability, proliferation, and death of hUCB-MSCs after NLRP3 inflammasome activation. (A) Expressions of NLRP3, ASC, pro or cleaved caspase-1, pro or mature IL-1 $\beta$ were analyzed by immunoblotting in hUCBMSCs after treatment with LPS, ATP or LPS + ATP. (B) The concentration of mature IL-1 $\beta$ in the culture supernatant of hUCB-MSCs was measured by ELISA. (C) Cell viability was detected by CCK-8. (D) Cell proliferation was determined by CPDL analysis. (E) Cell death was assessed by Live/Dead staining. Bar, $500 \mu \mathrm{m}$. Results show 1 representative experiment of at least 3 independent experiments. Results are shown as mean \pm SD.

\section{NLRP3 inflammasome stimulation does not alter the} proliferation and surface marker expression of hUCB-MSCs Pyroptosis is a caspase-1-mediated cell death that commonly occurs after inflammasome activation in macrophages and dendritic cells. Therefore, we investigated whether the viability or proliferation of hUCB-MSCs is affected by NLRP3 inflammasome activation-mediated pyroptosis. NLRP3 activation did not affect the viability, proliferation, and death of hUCB-MSCs (Fig. 1C-E). Next, the surface marker expression in naïve and activatedhUCB-MSCs was analyzed. The expression pattern of all negative (CD14, CD31, CD34, and CD45) or positive (CD29, CD44, and CD73) markers characterizing MSCs was not altered after NLRP3 activation (Supplementary Table. 1, Supplementary Fig. 1A and $1 \mathrm{~B})$. Our results imply that NLRP3 inflammasome activation in hUCB-MSCs neither induces pyroptosis nor affects the surface marker expression.

\section{NLRP3 activation inhibits the osteogenic differentiation of hUCB-MSCs}

Several studies have shown that differentiation of MSCs can be modulated by triggering of innate immune receptors. Therefore, the differentiation capability of hUCB-MSCs into osteoblasts or adipocytes was determined after NLRP3 inflammasome activa- 
A
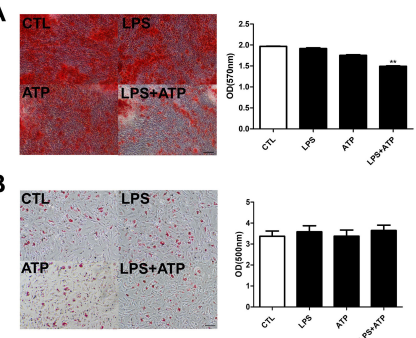

C

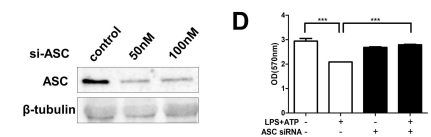

Fig. 2. Osteogenic and adipogenic differentiation of NLRP3-activated hUCB-MSCs. (A) hUCB-MSCs were cultured in osteoblast induction media and stained with Alizarin Red S. Stain was eluted and the absorbance was measured at $570 \mathrm{~nm}$. Bar, $250 \mu \mathrm{m}$. (B) hUCBMSCs were differentiated into adipocytes and stained by Oil Red O. Stain was eluted and the absorbance was measured at 500 nm. Bar, $250 \mu \mathrm{m}$. (C) siRNA for ASC was transfected to hUCB-MSCs and a decrease in protein level was observed. (D) siRNA-treated hUCB-MSCs were induced for osteogenic differentiation. The eluted stain was quantified. Results show 1 representative experiment of at least 3 independent experiments. Results are shown as mean \pm SD. $* * P<.01, * * * P<.001$

tion. We observed that osteogenic differentiation was significantly down-regulated upon NLRP3 inflammasome activation compared with naïve hUCB-MSCs (Fig. 2A). On the other hand, NLRP3 inflammasome activation did not exert any influence on the potential of adipogenic differentiation (Fig. 2B). To confirm that this inhibitory effect of NLRP3 inflammasome activation on osteogenic differentiation is mediated via inflammasome assembly, siRNA targeting ASC, an essential component for inflammasome assembly, was employed (Fig. 2C). Interestingly, ASC suppression restored the inhibitory effect of NLRP3 inflammasome activation on osteogenic differentiation (Fig. $2 \mathrm{D})$, suggesting that NLRP3 inflammasome is a negative regulator of osteogenesis in hUCB-MSCs.

\section{NLRP3 inflammasome activation augments the immunosuppressive properties of hUCB-MSCs}

MSCs have been reported to regulate the proliferation, activation, and maturation of various immune cells. Therefore, we evaluated in vitro immunosuppressive effects of hUCB-MSCs after NLRP3 inflammasome activation. PBMCs were co-cultured with naïve or activated-MSCs with concanavalin A or antiCD3/CD28. NLRP3 activation enhanced the suppressive effect of hUCB-MSCs on mitogen-induced PBMC proliferation or anti-CD3/CD28-induced T cell proliferation (Fig. 3A and 3B). To determine whether NLRP3 inflammasome activation influences the differentiation of helper T (Th) cells, Th cell-type-specific cytokine production in co-culture media was measured. Th1 and Th2 cell differentiation were assessed by detecting

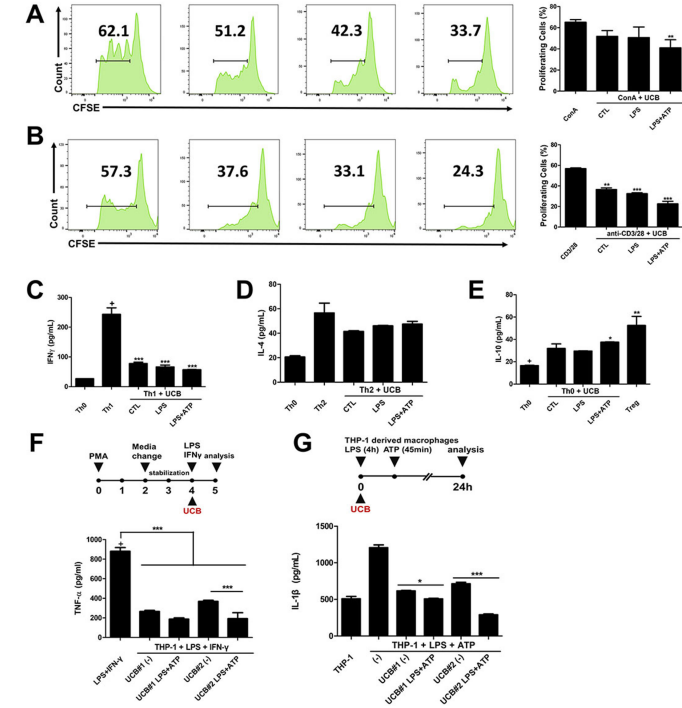

Fig. 3. Immunoregulatory properties of NLRP3-stimulated hUCB-MSCs. (A, B) PBMCs were stained with CFSE and then activated by (A) concanavalin $\mathrm{A}$ or (B) anti-CD3/28 to induce the proliferation of pan leukocytes or $\mathrm{T}$ cells. (C-E) $\mathrm{CD}^{+} \mathrm{T}$ cells isolated from PBMCs were co-cultured with hUCB-MSCs. (C, D) Th1 and Th2 differentiation was induced in the presence of hUCB-MSCs. (C) IFN- $\gamma$ and (D) IL-4 productions were measured. (E) Naïve $\mathrm{CD}^{+}{ }^{+} \mathrm{T}$ cells were $\mathrm{CO}^{-}$ cultured with hUCB-MSCs and IL-10 production was measured. (F) THP-1-derived M1 macrophages were co-cultured with hUCB-MSCs. TNF- $\alpha$ concentration in the culture media was detected. (G) NLRP3activated THP-1-derived macrophages were co-cultured with hUCBMSCs. IL-1 $\beta$ level in the culture media was detected. Results show 1 representative experiment of at least 3 independent experiments. Results are shown as mean $\pm \mathrm{SD}$. ${ }^{*} \mathrm{P}<.05,{ }^{*} * \mathrm{P}<.01,{ }^{* * * P}<.001$.

IFN- $\gamma$ and IL-4 production, respectively. Both naïve and activated hUCB-MSCs significantly reduced IFN- $\gamma$ production from Th1 cells (Fig. 3C). Although naïve or activated MSCs slightly inhibited IL-4 production from Th2 cells, the effect was not significant (Fig. 3D). To assess the ability of MSCs to induce regulatory $\mathrm{T}$ (Treg) cells, Th0 cells were co-cultured with MSCs without any differentiation induction. Interestingly, IL-10 production from $\mathrm{T}$ lymphocytes was significantly elevated when they were co-cultured with activated MSCs (Fig. 3E).

Given that MSCs can regulate macrophage activation, we next determined the regulatory function of hUCB-MSCs on M1 activation or NLRP3 activation of macrophages using THP-1derived macrophage-like cells. When THP-1-derived M1 macrophages were co-cultured with NLRP3-activated hUCB-MSCs, TNF- $\alpha$ production was down-regulated to a greater extent compared to naïve MSC addition (Fig. 3F). THP-1-derived macrophages produced increased IL-1 $\beta$ in response to LPS and ATP treatment, and co-culture with hUCB-MSCs suppressed IL-1 $\beta$ production from macrophages. Interestingly, activated hUCB-MSCs down-regulated IL-1 $\beta$ production to a significantly greater extent (Fig. 3G). Taken together, these results suggest 


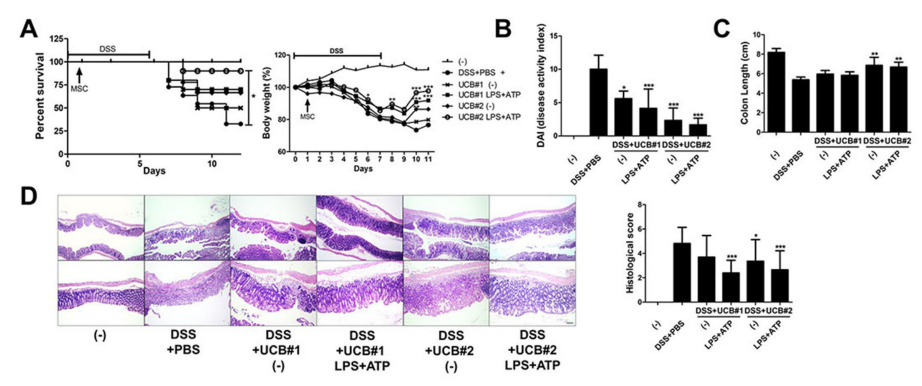

Fig. 4. Protective efficacy of NLRP3-stimulated hUCB-MSCs in the DSS-induced colitis model. DSS-induced colitic mice were injected with hUCB-MSCs. (A) Mice were monitored for survival rate and body weight loss. The statistical significance was calculated by comparing other groups against 'DSS+PBS' group (marked as '+'). (B) On day 10, the disease activity index was determined. (C) After sacrifice on day 12, colon length was measured. (D) Colon sections were prepared and stained with H\&E. Histopathologic evaluation was performed by determining the destruction of epithelial structure and lymphocyte infiltration. Bar, $500 \mu \mathrm{m}$. Results are shown as mean $\pm \mathrm{SD}$. *P $<.05, * * \mathrm{P}<.01, * * * \mathrm{P}<.001$.

that NLRP3 inflammasome activation in hUCB-MSCs generally augments the immunoregulatory abilities including suppressive effects on T cell proliferation, Th1 cell differentiation and macrophage activation, as well as facilitating effects on Treg generation.

\section{NLRP3 inflammasome activation improves the protective} efficacy of hUCB-MSCs against DSS-induced murine colitis We then investigated whether NLRP3 activation in hUCBMSCs can lead to any beneficial effects in vivo. To this aim, the DSS-induced murine colitis model was utilized as this colitic model involves $T$ cell- and macrophage-mediated immune responses in disease progression. DSS administration for one week resulted in typical symptoms of acute colitis. Intraperitoneal injection of hUCB-MSCs protected mice from lethality and body weight loss (Fig. 4A). Importantly, activated hUCBMSCs exerted greater protective effects compared to naïve MSCs. On day 10, the disease activity index was significantly decreased by cell infusion and further improved by NLRP3 inflammasome activation (Fig. 4B). Gross findings after necropsy demonstrated that the colon length was significantly restored by hUCB-MSC from one donor (UCB\#2, Fig. 4C). Upon histologic examination, the destruction of the entire epithelium and scattered infiltration of inflammatory cells were observed in the colon of DSS-treated mice. In hUCB-MSC injected mice, mucosal destruction and lymphocyte infiltration were reduced (Fig. 4D). Our findings demonstrate that NLRP3 inflammasome activation can augment the protective efficacy of hUCB-MSCs in vivo, consistent with in vitro anti-inflammatory abilities.

\section{NLRP3 activation does not affect the secretion of well-known} immunoregulatory soluble factors in hUCB-MSCs

MSCs have been reported to produce a variety of immunosuppressive soluble factors. To explore the key soluble factors in NLRP3 inflammasome activation, we measured more than 40 soluble factors in culture media from hUCB-MSCs. Based on the analysis of cytokine array, although there were slightly up-regulated factors including ALCAM and SDF- $1 \alpha$ upon NLRP3 activation (Supplementary Fig. 2A and 2B), their mRNA expression after treatment with LPS or LPS + ATP was not increased (Supplementary Fig. 3A). We also investigated the expression of recently reported physiological anti-inflammatory antagonists such as IL-1RA, IL-17RA, and IL-18BP. There were no significant differences between naïve and activated hUCB-MSCs regarding the expression of these antagonists at the mRNA level (Supplementary Fig. 3A). We previously suggested $\mathrm{PGE}_{2}$ as one of the major anti-inflammatory mediators for the immunomodulatory function of MSCs. However, although LPS treatment slightly increased the secretion of $\mathrm{PGE}_{2}, \mathrm{NLRP} 3$ inflammasome activation by LPS + ATP treatment did not affect $\mathrm{PGE}_{2}$ production (Supplementary Fig. 3B). These findings propose that NLRP3 inflammasome activation potentiates the immunoregulatory functions of hUCB-MSCs presumably through the regulation of novel paracrine factors or other cell functions, which have not been reported previously.

\section{DISCUSSION}

The assembly of NLRP3 inflammasome leads to the secretion of IL-1 $\beta$, followed by pyroptosis, an inflammatory programmed cell death by the microbial pathogens (15). However, in the present study, we could not observe the pyroptosis or significant production of IL-1 $\beta$ in MSC in response to NLRP3 inflammasome stimulation. Although the final phenotypes of inflammasome activation were not induced, osteogenic differentiation and immunomodulation of hUCB-MSCs were altered by NLRP3 inflammasome activation and were not affected by a single treatment with LPS or ATP. Several previous studies have shown that inflammasome or its components can regulate cell functions without classical activation. Wang et al. reported that NLRP3 can promote epithelial-mesenchymal transition in colon cancer cells in an inflammasome-independent manner (37). In addition, Kim et al. showed that hypoxia increased the expression of NLRP3 without IL-1 $\beta$ production 
in renal tubular cells to regulate the production of mitochondrial ROS (38). Moreover, NLRP3 has been suggested as a transcriptional regulator in Th2 differentiation by binding to IL4 promoter (39).

Here we have demonstrated that the osteogenic differentiation of hUCB-MSCs was impeded when NLRP3 inflammasome was activated. A recent study by Wang et al. reported that human umbilical cord-derived MSCs expressed the components of NLRP3 inflammasome and that its activation inhibited osteogenic differentiation while adipogenic differentiation was enhanced (36). It was consistently observed that NLRP3 inflammasome activation suppressed the osteogenic differentiation of hUCB-MSCs, which was restored by the inhibition of ASC expression. However, adipogenesis was not enhanced in the present study and this discrepancy might be due to the difference between the stimulators employed for inflammasome activation. In the present study, ATP was used for second step activation of NLRP3 inflammasome whereas palmitic acid was used in the previous study.

More importantly, in the present study, for the first time, we demonstrated that NLRP3 inflammasome activation in hUCBMSCs led to an increase in the immunosuppressive effects. Some studies reported the influence of PRR triggering on the immunomodulation of MSCs. In one study, TLR activation enhanced the immunosuppressive ability of hMSC by inducing an enzyme responsible for tryptophan catabolism (40). Also, Waterman et al. observed that TLR4-activated hMSCs secrete pro-inflammatory mediators, while TLR3-activated hMSCs efficiently suppress inflammation (41). Moreover, we previously demonstrated that NOD2 receptor stimulation can efficiently enhance the anti-inflammatory functions of hUCB-MSCs (33, 34). In this study, NLRP3 inflammasome activation augmented the protective efficacy of hUCB-MSCs in vivo, as well as in vitro anti-inflammatory abilities in several immune cells including Th cells and macrophages. This result supports the possibility that MSCs could be activated in the presence of signals for NLRP3 inflammasome activation and that robust stimulation of MSCs leads to physiologically sufficient immunoregulatory effects. The limitation of our study is that crucial paracrine factors could not be discovered although various screening assays were performed for already known major immunomodulatory factors. In this respect, it is apparent that future work will require the precise elucidation of the underlying mechanism for improved function of NLRP3 inflammasome activated-MSCs by uncovering pivotal soluble factors.

In conclusion, our findings clarified that NLRP3 stimulation decreased the differentiation potential of hUCB-MSCs into osteoblasts and increased immunosuppressive effects. We anticipate that these findings could provide a better understanding of the inflammasome-mediated regulation of non-immune cell functions, as well as a basis for the development of highly efficient cell therapy to treat several intractable inflammatory diseases.

\section{MATERIALS AND METHODS}

The detailed methods are described in the "Supplementary Materials and Methods".

\section{ACKNOWLEDGEMENTS}

This research was supported by the National Research Foundation of Korea (NRF) grant funded by the Korea government (MSIT) (No. NRF-2016R1C1B2016140, NRF-2018R1A5A2023 879, and NRF-2018R1D1A1B07048035). We would like to thank Kangstem Biotech for providing hUCB-MSCs.

\section{CONFLICTS OF INTEREST}

The authors have no conflicting interests.

\section{REFERENCES}

1. Akira S, Uematsu $S$ and Takeuchi $O$ (2006) Pathogen recognition and innate immunity. Cell 124, 783-801

2. Beutler B, Eidenschenk C, Crozat K et al (2007) Genetic analysis of resistance to viral infection. Nat Rev Immunol 7, 753-766

3. Hoffmann JA (2003) The immune response of Drosophila. Nature 426, 33-38

4. Medzhitov R (2007) Recognition of microorganisms and activation of the immune response. Nature 449, 819-826

5. Kato H, Takahasi K and Fujita T (2011) RIG-I-like receptors: cytoplasmic sensors for non-self RNA. Immunol Rev 243, 91-98

6. Kawai T and Akira S (2011) Toll-like receptors and their crosstalk with other innate receptors in infection and immunity. Immunity 34, 637-650

7. Janeway CA Jr (1989) Approaching the asymptote? Evolution and revolution in immunology. Cold Spring Harb Symp Quant Biol 54 Pt 1, 1-13

8. van den Berk LC, Jansen BJ, Siebers-Vermeulen KG et al (2009) Toll-like receptor triggering in cord blood mesenchymal stem cells. J Cell Mol Med 13, 3415-3426

9. Tomchuck SL, Zwezdaryk KJ, Coffelt SB, Waterman RS, Danka ES and Scandurro AB (2008) Toll-like receptors on human mesenchymal stem cells drive their migration and immunomodulating responses. Stem Cells 26, 99-107

10. Lombardo E, DelaRosa O, Mancheno-Corvo P, Menta R, Ramirez C and Buscher D (2009) Toll-like receptor-mediated signaling in human adipose-derived stem cells: implications for immunogenicity and immunosuppressive potential. Tissue Eng Part A 15, 1579-1589

11. Kim HS, Shin TH, Yang SR et al (2010) Implication of NOD1 and NOD2 for the differentiation of multipotent mesenchymal stem cells derived from human umbilical cord blood. PLoS One 5, e15369

12. Hwa Cho H, Bae YC and Jung JS (2006) Role of toll-like receptors on human adipose-derived stromal cells. Stem Cells 24, 2744-2752

13. Vajjhala PR, Mirams RE and Hill JM (2012) Multiple bin- 
ding sites on the pyrin domain of ASC protein allow selfassociation and interaction with NLRP3 protein. J Biol Chem 287, 41732-41743

14. Martina P, Motti G, Peter DM, John CR and Stefan JR (2013) The CARD plays a critical role in ASC foci formation and inflammasome signalling. Biochem J 449, 613- 621

15. Broderick L, De Nardo D, Franklin BS, Hoffman HM and Latz E (2015) The inflammasomes and autoinflammatory syndromes. Ann Rev Pathol 10, 395-424

16. Bauernfeind FG, Horvath G, Stutz A et al (2009) Cutting edge: NF- $\mathrm{BB}$ activating pattern recognition and cytokine receptors license NLRP3 inflammasome activation by regulating NLRP3 expression. J Immunol 183, 787-791

17. Yu J, Nagasu H, Murakami T et al (2014) Inflammasome activation leads to Caspase-1-dependent mitochondrial damage and block of mitophagy. Proc Natl Acad Sci U S A 111, 15514-15519

18. Kim SM, Ha JS, Han AR, Cho SW and Yang SJ (2019) Effects of $\alpha$-lipoic acid on LPS-induced neuroinflammation and NLRP3 inflammasome activation through the regulation of BV-2 microglial cells activation. BMB Rep 52, 613-618

19. Augello A, Tasso R, Negrini SM, Cancedda R and Pennesi G (2007) Cell therapy using allogeneic bone marrow mesenchymal stem cells prevents tissue damage in collagen induced arthritis. Arthritis Rheum 56, 1175-1186

20. Gonzalez MA, Gonzalez-Rey E, Rico L, Buscher D and Delgado M (2009) Adipose-derived mesenchymal stem cells alleviate experimental colitis by inhibiting inflammatory and autoimmune responses. Gastroenterology 136, 978-989

21. Le Blanc K, Rasmusson I, Sundberg B et al (2004) Treatment of severe acute graft-versus-host disease with third party haploidentical mesenchymal stem cells. Lancet 363, 1439-1441

22. Lee RH, Seo MJ, Reger RL et al (2006) Multipotent stromal cells from human marrow home to and promote repair of pancreatic islets and renal glomeruli in diabetic NOD/scid mice. Proc Natl Acad Sci U S A 103, 17438

23. Nemeth K, Leelahavanichkul A, Yuen PST et al (2008) Bone marrow stromal cells attenuate sepsis via prostaglandin E2-dependent reprogramming of host macrophages to increase their interleukin-10 production. Nat Med 15, 4249

24. Zappia E, Casazza S, Pedemonte E et al (2005) Mesenchymal stem cells ameliorate experimental autoimmune encephalomyelitis inducing T-cell anergy. Blood 106, 1755

25. Kang I, Lee BC, Lee JY et al (2019) Interferon- $\gamma$-mediated secretion of tryptophanyl-tRNA synthetases has a role in protection of human umbilical cord blood-derived mesenchymal stem cells against experimental colitis. BMB Rep 52, 318-323

26. Ren G, Zhang L, Zhao X et al (2008) Mesenchymal stem cell-mediated immunosuppression occurs via concerted action of chemokines and nitric oxide. Cell Stem Cell 2, $141-150$

27. Krampera M, Glennie S, Dyson J et al (2003) Bone marrow mesenchymal stem cells inhibit the response of naive and memory antigen-specific T cells to their cognate peptide. Blood 101, 3722

28. Beyth S, Borovsky Z, Mevorach D et al (2005) Human mesenchymal stem cells alter antigen-presenting cell maturation and induce T-cell unresponsiveness. Blood 105, 2214

29. Puissant B, Barreau C, Bourin P et al (2005) Immunomodulatory effect of human adipose tissue derived adult stem cells: comparison with bone marrow mesenchymal stem cells. Br J Haematol 129, 118-129

30. Yanez R, Lamana ML, Garcia Castro J, Colmenero I, Ramirez M and Bueren JA (2006) Adipose Tissue Derived Mesenchymal Stem Cells Have In Vivo Immunosuppressive Properties Applicable for the Control of the Graft Versus Host Disease. Stem Cells 24, 2582-2591

31. Sato K, Ozaki K, Oh I et al (2007) Nitric oxide plays a critical role in suppression of T-cell proliferation by mesenchymal stem cells. Blood 109, 228

32. Tse WT, Pendleton JD, Beyer WM, Egalka MC and Guinan EC (2003) Suppression of allogeneic T-cell proliferation by human marrow stromal cells: implications in transplantation. Transplantation 75, 389

33. Kim HS, Shin TH, Lee BC et al (2013) Human umbilical cord blood mesenchymal stem cells reduce colitis in mice by activating NOD2 signaling to COX2. Gastroenterology 145, 1392-1403 e1391-1398

34. Kim HS, Yun JW, Shin TH et al (2015) Human umbilical cord blood mesenchymal stem cell-derived PGE2 and TGF-beta1 alleviate atopic dermatitis by reducing mast cell degranulation. Stem Cells 33, 1254-1266

35. Oh JY, Ko JH, Lee HJ et al (2014) Mesenchymal stem/stromal cells inhibit the NLRP3 inflammasome by decreasing mitochondrial reactive oxygen species. Stem Cells 32, 1553-1563

36. Wang L, Chen K, Wan X, Wang F, Guo Z and Mo Z (2017) NLRP3 inflammasome activation in mesenchymal stem cells inhibits osteogenic differentiation and enhances adipogenic differentiation. Biochem Biophys Res Commun 484, 871-877

37. Wang H, Wang $Y$, Du $Q$ et al (2016) Inflammasomeindependent NLRP3 is required for epithelial-mesenchymal transition in colon cancer cells. Exp Cell Res 342, 184-192

38. Kim SM, Kim YG, Kim DJ et al (2018) InflammasomeIndependent Role of NLRP3 Mediates Mitochondrial Regulation in Renal Injury. Front Immunol 9, 2563

39. Bruchard M, Rebe C, Derangere V et al (2015) The receptor NLRP3 is a transcriptional regulator of $\mathrm{TH} 2$ differentiation. Nat Immunol 16, 859-870

40. Opitz CA, Litzenburger UM, Lutz C et al (2009) Toll-like receptor engagement enhances the immunosuppressive properties of human bone marrow-derived mesenchymal stem cells by inducing indoleamine-2,3-dioxygenase- 1 via interferon-beta and protein kinase R. Stem Cells 27, 909919

41. Waterman RS, Tomchuck SL, Henkle SL and Betancourt AM (2010) A new mesenchymal stem cell (MSC) paradigm: polarization into a pro-inflammatory MSC1 or an Immunosuppressive MSC2 phenotype. PLoS One 5, e10088 